Pacific Journal of Mathematics

OFF DIAGONAL ASYMPTOTICS OF HYPOELLIPTIC
DIFFUSION EQUATIONS AND SINGULAR RIEMANNIAN

OM JOSEPH TAYLOR 


\title{
OFF DIAGONAL ASYMPTOTICS OF HYPOELLIPTIC DIFFUSION EQUATIONS AND SINGULAR RIEMANNIAN GEOMETRY
}

\author{
THOMAS J. S. TAYLOR
}

The major purpose of this paper is to investigate some small time asymptotic properties of certain diffusion equations of the form

$$
\left(\frac{\partial}{\partial t}-\Delta\right) \rho(y, x, t)=0, \quad \lim _{t \rightarrow 0} \rho(y, x, t)=\delta_{x}(y),
$$

where $\Delta$ is in a class of second order hypoelliptic differential operators on a connected $m$-dimensional manifold $M$, and where $\delta_{x}(y)$ is the Dirac $\delta$-function in the variable $y \in M$ supported at the point $x \in M$.

0. Introduction. We assume for the duration of this paper that $\Delta$ is locally of the form $\Delta=\sum_{a=1}^{n} g_{a}^{2}+g_{0}$ where $\left\{g_{a}\right\}_{a=0}^{n}$ are smooth vector fields which satisfy the condition that the Lie algebra generated (over $\mathbf{R}$ ) by the vector fields $\left\{g_{a}\right\}_{a=1}^{n}$ has the property that at each point $x \in M$ the evaluation map at $x$ maps this Lie algebra onto the tangent space $T_{x} M$. This condition is (more than) enough to imply that $\rho(y, x, t)$ is a smooth function of all variables for $t>0$, by a theorem of Hörmander [13].

A major tool I will use in this investigation will be aspects of differential geometry as developed by Gromov [11] and in the paper [25] by Taylor, as well as various references contained therein, and the transmutation theory developed in the paper of Kannai [17].

The basic thrust of this paper is that, given that certain geometric conditions hold, "off the diagonal" $\rho(y, x, t)$ behaves (generically) very much like the solutions of parabolic diffusion equations. In particular, we obtain for a certain class of hypoelliptic operators an asymptotic expansion of the fundamental solution; this implies that a formula first derived by Varadhan [26] in the case that $\Delta$ is an elliptic operator

$$
\lim _{t \rightarrow 0}-4 t \ln \rho(y, x, t)=r(x, y)^{2},
$$

holds also in the hypoelliptic case where $r(y, x)$ is the distance function discussed by Brockett [4], Gromov [11], Strichartz [22] and Taylor [25], and is the "subelliptic metric" discussed by Sanchez-Calle [21] and Fefferman and Phong [9]. This result has been obtained in the 
special case that $\Delta$ is the subelliptic Laplacian on the Heisenberg group by Gaveau [10] and by Azencott [2]. Azencott has also proven certain inequalities related to equation (2) using large deviation methods. Bismut [3] has discussed this problem, also from a stochastic point of view, using large deviations and the Malliavin calculus. Menikoff and Sjostrand [19] have constructed the kernel $\rho(y, x, t)$ mod smooth functions which vanish to infinite order at $t=0$, but this is not enough to imply formula (2).

My method of proving this result is to follow closely the method developed by Kannai [17] for obtaining asymptotics of parabolic diffusion equations, using results of differential geometry of Gromov [11] and Taylor [25] which generalize the aspects of Riemannian geometry used by Kannai. In particular, I need to study the wave front set and support of solutions of the wave equation

$$
\left(\frac{\partial^{2}}{\partial s^{2}}-\Delta\right) W=0, \quad W(y, x, 0)=\delta_{x}(y), \quad W_{t}(y, x, 0)=0 .
$$

Because $\Delta$ is not an elliptic operator, equation (3) is not of real principle type, so that the issue of the wave front set is not as simple an issue as the situation discussed by Kannai [17]. In particular, Kannai's method has clear application only in the case that the wave front set has no doubly characteristic points over certain subsets. The state of the art in the propagation of singularities literature for this equation seems to be (in the paper of Lascar and Lascar [18]) that we can guarantee this property when the double characteristics of $\Delta$ form a symplectic submanifold of $T^{*} M$.

Interestingly, this condition appears also in Bismut [3] as a sufficient condition for invertibility of the Malliavin covariance matrix, as well as in the partial differential equations literature.

In order to apply the paper of Kannai and the paper of Taylor to study this situation, I need to make some assumptions. The first is that the bicharacteristic flow of $\Delta$ is complete and that for every two points $x, y \in M$ there is a bicharacteristic $\gamma:[0,1] \rightarrow T^{*} M$ such that $\pi \gamma(0)=x$ and $\pi \gamma(1)=y$, where $\pi$ is the canonical projection of $T^{*} M$ on $M$; see [25] for a discussion of these conditions. This will guarantee finite speed of propagation but places certain restrictions on the operators allowed. A second assumption is that $\Delta$ is symmetric with respect to a smooth measure. This also constrains the class of operators considered. As suggested above, we also assume that the double characteristics of $\Delta$ form a symplectic submanifold; besides the 
implications for the wavefront set, this will have certain geometrical implications which allow us to apply the results of [25].

Now, let $D \subset T M$ denote the distribution spanned at each $x \in M$ by the tangent vectors $\left\{g_{a}(x)\right\}_{a=1}^{n}$. Let $D^{\perp} \subset T^{*} M$ denote the annihilator of $D$. We have the conditions:

(4a) $D^{\perp}$ is a rank one subbundle of $T^{*} M$.

(4b) $D$ is a rank $n$ subbundle of $T M$.

(4c) For each $x \in M$ and $0 \neq \eta_{x} \in D^{\perp}$, the form given on $D_{x} \times D_{x}$ by the linear extension of $\left(g_{a}(x), g_{b}(x)\right) \mapsto\left\langle\eta,\left[g_{a}, g_{b}\right](x)\right\rangle$ is of $\operatorname{rank} n=2 q$.

It follows from (4) that the doubly characteristic set $\sum=\bigcap_{a=1}^{n}\left\{g_{a}=\right.$ $0\}=D^{\perp}$ is a symplectic submanifold of $T^{*} M$ (we consider $g_{a}$ as a function on $T^{*} M$, linear on the fibers). See Lascar and Lascar [18] and M. Taylor [23] for relevant discussion. Note that conditions (4a) and (4b) imply that $\operatorname{dim}(M)=2 q+1$, while condition (4c) implies that the Lie algebra generated by $\left\{g_{a}\right\}_{a=1}^{n}$ spans at step two.

In $\S 1$, I discuss some geometrical aspects of selfadjoint second order hypoelliptic operators, in the light of the singular Riemannian geometry discussed in [25]. I recall some properties of the wave equation (3) associated with a self adjoint hypoelliptic operator $\Delta . W(y, x, t)$ is the Schwartz kernel of the operator $\cos (t \sqrt{-\Delta})$ discussed in [4] for the case that $\Delta$ is elliptic and in [23] for some hypoelliptic cases. In particular, I look at the transmutation formula

$$
\rho(y, x, t)=\int_{-\infty}^{\infty} \frac{e^{-s^{2} / 4 t}}{\sqrt{4 \pi t}} W(y, x, s) d s
$$

which transforms solutions of (3) into solutions of (1). In $\S 2, I$ apply the propagation of singularities theory developed by Hörmander [13] and Duistermaat and Hörmander [8] to the wave equation (3). The book of Guillemin and Sternberg is also helpful here [12]. The only thing new about this is that the results of [25] allow me to describe more accurately than before the simply characteristic wavefront set of $W$.

The doubly characteristic wavefront set of solutions of various equations of the form (3) have been discussed in the literature, for example by Ivrii [16], Lascar and Lascar [18], and M. Taylor [23]. The state of the art seems to be the paper of Lascar and Lascar, which considers our situation of a symplectic doubly characteristic set. It follows that over a certain region in the support of $W$, the wavefront set of $W$ contains no doubly characteristic points. From this we obtain an 
expansion

$$
W(y, x, t) \sim \sum_{b=(1-m) / 2}^{\infty} \delta^{(-k)}\left(t^{2}-r(x, y)^{2}\right) c_{k}(x, y)
$$

when $(y, x, t)$ is in a certain open subset of $M \times M \times R$, where $r(y, x)$ is again the singular Riemannian distance function. I then apply the recipe of Kannai to this formula to obtain (Theorem 2)

$$
\rho(y, x, t) \sim \frac{e^{-r(y, x)^{2} / 4 t}}{(4 \pi t)^{m / 2}} \sum_{k=0}^{\infty} v_{k}(x, y) t^{k}
$$

for $(y, x)$ in a certain open dense subset of $M \times M$.

This result may then be combined with the Chapman-Kolmogorov formula (as discussed by Kannai) to prove the result that for all $(x, y) \in$ $M \times M$ :

$$
\lim _{t \rightarrow 0}-4 t \ln \rho(y, x, t)=r(y, x)^{2} .
$$

The discussion of this paper should be compared with Theorem II of Sanchez-Calle [21].

One should note the paper of Cheeger, Gromov and Taylor [4], which contains some ideas related to those of Kannai and uses Riemannian geometry quite deeply. Some of the results of that paper should generalize to the situation discussed here, to the extent that the Riemannian geometry used has analogs in the singular Riemannian geometry.

It should be clear by this point that this work is very much influenced by the paper of Kannai; it seems to me that the techniques developed there ought to have many other applications.

1. Geometrical aspects. In this section, I discuss the construction of self adjoint hypoelliptic operators associated to a singular Riemannian structure. In this section, I will follow the notation of Taylor [25]. In particular, $G^{*}$ will denote the nonnegative, degenerate quadratic form or $T^{*} M$ determined by $\Delta, G$ will denote the dual quadratic form on the distribution $D \subset T M$ determined by $G^{*}, D^{\perp}=\operatorname{ker} G^{*}$ is the annihilator of $D$ in $T^{*} M$, and $r(y, x)$ denotes the singular Riemannian metric on $M$ determined by $G$. This construction does not depend on having the bundle $D$ being trivial. In this section let $\mu$ denote a smooth measure on $M$; i.e., $\mu$ restricted to any coordinate chart has nonzero $C^{\infty}$ density with respect to Lebesgue measure there. Let $\pi$ denote the canonical projection of $T^{*} M$ onto $D^{*}=T^{*} M / \operatorname{ker}\left(G^{*}\right)$. Let $\tilde{G}^{*}$ denote the quadratic form on $D^{*}$ which comes from the $G^{*}$ on 
$T^{*} M$. Let $E_{c}(M)$ denote the space of compactly supported smooth sections of $D^{*} . E_{c}(M)$ is a pre-Hilbert space with respect to the inner product $\langle u, v\rangle^{\prime}=\int \tilde{G}^{*}(\bar{u}, v) d \mu(x)$; use the symbol $H(M)$ to denote the associated Hilbert space (all square integrable sections). Now, let $d$ denote the de Rham operator taking smooth functions on $M$ to 1forms. Then, $\pi d$ is a differential operator from compactly supported smooth functions to $E_{c}(M)$. Let $\delta: L^{2}(M, d \mu) \rightarrow H(M)$ be the maximal closed extension of the operator $\pi d$; i.e., the operator $\pi d$ with domain $\mathscr{D}(\delta)=\left\{f \in L^{2}(M)\right.$ : in the distributional sense, $\pi d f$ is in $H(M)\}$. Then, the operator $\Delta=-\delta^{*} \delta$ is a self adjoint unbounded operator on $L^{2}(M)$.

Suppose that $\left\{U_{\alpha}\right\}$ is a countable open cover for $M$ and that for each $\alpha, D \mid U_{\alpha}$ has an orthonormal trivialization $\left\{g_{a}^{\alpha}\right\}$. Suppose also that $\left\{\phi_{\alpha}\right\}$ is a partition of unity subordinate to the cover $\left\{U_{\alpha}\right\}$. Then, the operator $\Delta$, is given on $C_{0}^{\infty}$ by

$$
\Delta f=\sum_{\alpha} \phi_{\alpha} \sum_{a=1}^{n}\left(g_{a}^{\alpha} g_{a}^{\alpha}+\operatorname{div}_{\mu}\left(g_{a}^{\alpha}\right) g_{a}^{\alpha}\right) f .
$$

Thus, $\Delta$ is a self adjoint operator which may be represented in local terms as a sum of vector fields and squares of vector fields. Moreover, because of the fact that $T M$ is the smallest integrable distribution which contains $D$, the span of the Lie algebra of vector fields generated by $\left\{g_{a}^{\alpha}\right\}$ is $T U_{\alpha}$ so that the Hörmander condition is satisfied, so that $\Delta$ is a hypoelliptic operator.

We have, in addition, that the principal symbol of $\Delta$ is just the function $G_{x}^{*}(p, p)$ for $(x, p) \in T^{*} M$, since the symbol of $\Delta$ is just $\sum_{\alpha} \phi_{\alpha} \sum_{a=1}^{n} g_{a}^{\alpha} \otimes g_{a}^{\alpha}$. We also have that $\Delta$ is a non-positive operator, in the sense that $\langle f, \Delta f\rangle \leq 0$ for $f \in \mathscr{D}(\Delta)$.

Consider the partial differential equation

$$
\left(\frac{\partial^{2}}{\partial t^{2}}-\Delta\right) W(t, x)=0
$$

In the following sections, I will consider this equation primarily as a weak partial differential equation on the space of distributions; in this section, I consider it as an evolution equation on suitable Hilbert spaces following standard methods for the case that $\Delta$ is elliptic. Now, equation (3) is equivalent to the equation

$$
\frac{\partial}{\partial t}\left[\begin{array}{l}
W \\
W_{t}
\end{array}\right]=\left[\begin{array}{ll}
0 & I \\
\Delta & 0
\end{array}\right]\left[\begin{array}{l}
W \\
W_{t}
\end{array}\right] .
$$


We consider $T=\left[\begin{array}{ll}0 & I \\ \Delta & 0\end{array}\right]$ as an unbounded operator on the space $H_{0}=$ $\mathscr{D}\left([-\Delta]^{1 / 2}\right) \oplus L^{2}(M)$, which is a Hilbert space with respect to the usual "energy" inner product defined by:

$$
\langle\cdot, \cdot\rangle_{0}=\left\langle[-\Delta]^{1 / 2} \cdot,[-\Delta]^{1 / 2} \cdot\right\rangle+\langle\cdot, \cdot\rangle .
$$

One can show that $T$ is anti-self adjoint on the domain $\mathscr{D}(\Delta) \oplus$ $\mathscr{D}\left([-\Delta]^{1 / 2}\right)$. Thus, $T$ generates a strongly continuous unitary group on $H_{0}$; call it $U(t)$.

REMARK. Of course, this requires that the spectrum of $\Delta$ is bounded away from zero. Since $\Delta$ is nonpositive $\Delta-1$ is bounded away from zero and if $(\partial / \partial t-\Delta+1) V=0$ then $(\partial / \partial t-\Delta) e^{t} V=0$, while multiplication by $e^{t}$ changes the asymptotics of $V$ in an obvious way. Thus, there is no loss of generality in assuming that the spectrum of $\Delta$ is bounded away from zero.

Now, the various powers $T^{q}$ of $T$ will be closed operators on the domain $\mathscr{D}\left(T^{q}\right)=\mathscr{D}\left([-\Delta]^{(q+1) / 2}\right) \oplus \mathscr{D}\left([-\Delta]^{q / 2}\right) ; T^{q}$ will be self adjoint if $q$ is even and anti-self adjoint when $q$ is odd.

$\mathscr{D}\left(T^{q}\right)$ can be made into a pre-Hilbert space with respect to the inner product $\langle\cdot, \cdot\rangle_{q}=\left\langle[-\Delta]^{q / 2} \cdot,[-\Delta]^{q / 2} \cdot\right\rangle_{0}$. Because $\Delta$ is a closed operator and bounded away from zero, it follows that $\mathscr{D}\left(T^{q}\right)$ is in fact a Hilbert space. The following lemma is well known:

LEMMA 3. $U(t) \mid \mathscr{D}\left(T^{q}\right)$ is a strongly continuous unitary group; its generator is $T \mid \mathscr{D}\left(T^{q+1}\right)$.

Now, since $\mathscr{D}\left(T^{q}\right)$ is a Hilbert space, there is a canonical isomorphism $\mathscr{D}\left(T^{q}\right) \cong \mathscr{D}\left(T^{q}\right)^{*}$. However, as is standard, we wish to forget for the moment this identification and consider $\mathscr{D}\left(T^{q}\right)$ to be properly included in $\mathscr{D}\left(T^{q}\right)^{*}$ via the inclusions

$$
\mathscr{D}\left(T^{q}\right) \rightarrow H_{0}(M)=H_{0}(M)^{*} \rightarrow \mathscr{D}\left(T^{q}\right)^{*} .
$$

The following lemma, due to Hörmander [13], will be sufficient for our needs, although Rothschild and Stein [20] have proven more precise results.

LEMMA 4. Suppose that $u, w$ are distributions and that $\Delta$ is a hypoelliptic operator of the type considered here. Then, for every open $\Omega \subset M$ of compact closure, there is an $\varepsilon>0$ such that if $\Delta u=w$, and $w \in L_{\text {loc }}^{r, 2}(\Omega)$ then $u \in L_{\text {loc }}^{r+\varepsilon, 2}(\Omega)$. 
COROLlaRY 5. For every open $\Omega \subset M$ of compact closure, there is an $\varepsilon>0$ such that for all even $q>0$

$$
f \in \mathscr{D}\left(T^{q}\right) \Rightarrow f \mid \Omega \in L_{\varepsilon q / 2, \mathrm{loc}}^{2}(\Omega) \oplus L_{\varepsilon q / 2, \mathrm{loc}}^{2}(\Omega)
$$

and

$$
f \in L_{-\varepsilon q / 2, \mathrm{loc}}^{2}(\Omega) \oplus L_{-\varepsilon q / 2, \mathrm{loc}}^{2}(\Omega) \Rightarrow \phi f \in \mathscr{D}\left(T^{q}\right)^{*},
$$

where $\phi$ is any element of $C_{0}^{\infty}(\Omega)$.

Corollary 6. $\bigcap_{q>0} \mathscr{D}\left(T^{q}\right) \subset C^{\infty}(M) \oplus C^{\infty}(M)$.

Thus, in particular, $\mathscr{D}\left(T^{q}\right)$ is a space of functions which are more smooth than $L^{2}$ and its dual is a space of distributions which are more singular than $L^{2}$.

If we now re-establish the identity between $\mathscr{D}\left(T^{q}\right)$ and $\mathscr{D}\left(T^{q}\right)^{*}$ we see that $\mathscr{D}\left(T^{q}\right)^{*}$ is a Hilbert space and that $U(t)$ extends to a strongly continuous unitary group on $\mathscr{D}\left(T^{q}\right)^{*}$. Moreover, the operator $S=$ $i[-\Delta]^{1 / 2}\left[\begin{array}{ll}1 & 0 \\ 0 & 1\end{array}\right]$ is a unitary operator considered as a mapping between

$$
\mathscr{D}\left(T^{q}\right)=\mathscr{D}\left([-\Delta]^{(q+1) / 2}\right) \oplus \mathscr{D}\left([-\Delta]^{q / 2}\right) \text { and } \mathscr{D}\left(T^{q-1}\right) .
$$

Since $S$ commutes with $T$ and with $U(t)$, it follows that $S$ extends to a unitary map of $H_{0}$ onto $\mathscr{D}(T)^{*}$ and more generally of $\mathscr{D}\left(T^{q}\right)^{*}$ onto $\mathscr{D}\left(T^{q+1}\right)^{*}$. One sees that the generator of $U(t)$ on $\mathscr{D}\left(T^{q}\right)^{*}$ is $T \mid \mathscr{D}\left(T^{q-1}\right)^{*}$ (since $S^{-1}$ maps $H_{0}$ onto $\mathscr{D}(T)$ ).

The following is a slight modification of a lemma of Kannai [17].

LEMMA 5. If the Cauchy data for equation (3) are in $\mathscr{D}\left(T^{q}\right)$ (resp. $\left.\mathscr{D}\left(T^{q}\right)^{*}\right)$ at $t=0$, then the integral

$$
V(t)=\int_{-\infty}^{\infty} \frac{e^{-s^{2} / 4 t}}{\sqrt{4 \pi t}} W(s) d s
$$

converges as a Bochner integral in $\mathscr{D}\left(\Delta^{(q+1) / 2}\right)$ (resp. $\mathscr{D}\left(\Delta^{(q+1) / 2}\right)^{*}$ and in fact is a $C^{\infty}$ function on $M \times\{t \in R: t>0\}$. Let $\phi \in C^{\infty}(R)$ be such that $\phi(t)=0$ for $t<1 / 2, \phi(t) \equiv 1$ for $t \geq 1$. Then, for every real number $a>0$ the function

$$
V(y, t ; a)=\int_{-\infty}^{\infty} \frac{\phi(|s|-a)}{\sqrt{4 \pi t}} e^{-s^{2} / 4 \pi t} W(s, y) d s
$$

is $C^{\infty}$ on $M \times\{t: t>0\}$ and satisfies the estimate $V(y, t, a)=$ $O_{t \rightarrow 0}\left(e^{-a^{2} / 4 t}\right)$ uniformly on compact subsets of $M$. 
Proof. Suppose that the Cauchy data $(\phi, \psi) \in \mathscr{D}\left(T^{q}\right)\left(\operatorname{resp} . \mathscr{D}\left(T^{q}\right)^{*}\right)$. Then, since $U(t)$ is unitary, the norm of $U(t)\left(\begin{array}{l}\phi \\ \psi\end{array}\right)$ in $\mathscr{D}\left(T^{q}\right)$ (resp. $\left.\mathscr{D}\left(T^{q}\right)^{*}\right)$ is independent of $t$, and since $U(t)$ is strongly continuous, $U(t)\left(\begin{array}{l}\phi \\ \psi\end{array}\right)$ is a bounded measurable function of $t$. Thus, for all $t>0$ the Bochner integral

$$
Y(t)=\int_{-\infty}^{\infty} \frac{e^{-s^{2} / 4 t}}{\sqrt{4 \pi t}}\left[\begin{array}{c}
W(s) \\
\frac{d}{d s} W(s)
\end{array}\right] d s
$$

converges in $\mathscr{D}\left(T^{q}\right)=\mathscr{D}\left(\Delta^{(q+1) / 2}\right) \oplus \mathscr{D}\left(\Delta^{q / 2}\right)$ uniformly with respect to $t$ (resp. in $\left.\mathscr{D}\left(T^{q}\right)^{*}=\mathscr{D}\left(\Delta^{(q+1) / 2}\right)^{*} \oplus \mathscr{D}\left(\Delta^{q / 2}\right)^{*}\right)$. Likewise, we have

$$
\begin{aligned}
\frac{\partial^{k}}{\partial t^{k}} Y(t) & =\frac{\partial^{k}}{\partial t^{k}} \int_{-\infty}^{\infty} \frac{e^{-s^{2} / 4 t}}{\sqrt{4 \pi t}}\left[\begin{array}{c}
W(s) \\
\frac{d}{d s} W(s)
\end{array}\right] d s \\
& =\int_{-\infty}^{\infty} \frac{\partial^{2 k}}{\partial s^{2 k}} \frac{e^{-s^{2} / 4 t}}{\sqrt{2 \pi t}}\left[\begin{array}{c}
W(s) \\
\frac{d}{d s} W(s)
\end{array}\right] d s
\end{aligned}
$$

is in $\mathscr{D}\left(T^{q}\right)$ (resp. $\mathscr{D}\left(T^{q}\right)^{*}$ ) for all $t>0$ (since the latter integral converges uniformly). At this point we can make use of the inclusions $\mathscr{D}\left(T^{q+2}\right) \rightarrow \mathscr{D}\left(T^{q}\right) \rightarrow L^{2} \rightarrow \mathscr{D}\left(T^{q}\right)^{*} \rightarrow \mathscr{D}\left(T^{q+2}\right)^{*}$ for all $q$ together with the fact that $S^{2}=-\left[\begin{array}{ll}\Delta & 0 \\ 0 & \Delta\end{array}\right]$ is a unitary map of $\mathscr{D}\left(T^{q+2}\right)$ onto $\mathscr{D}\left(T^{q}\right)$ to show that $\left[\begin{array}{c}W(s) \\ d W(s) / d s\end{array}\right]$ is $2 k$-times differentiable if we regard it to be a function, say, of $s$ into $\mathscr{D}\left(T^{q+2 k}\right)^{*}$. Then, we can integrate by parts to obtain

$$
\begin{aligned}
\frac{\partial^{k}}{\partial t^{k}} Y(t) & =\int_{-\infty}^{\infty} \frac{e^{-s^{2} / 4 t}}{\sqrt{4 \pi t}} \frac{\partial^{2 k}}{\partial s^{2 k}}\left[\begin{array}{c}
W(s) \\
\frac{\partial}{\partial s} W(s)
\end{array}\right] d s \\
& =\int_{-\infty}^{\infty} \frac{e^{-s^{2} / 4 t}}{\sqrt{4 \pi t}} \Delta^{k}\left[\begin{array}{c}
W(s) \\
\frac{\partial}{\partial s} W(s)
\end{array}\right] d s \\
& =T^{2 k} Y(t) \in \mathscr{D}\left(T^{q}\right)\left(\operatorname{resp.} \mathscr{D}\left(T^{q}\right)^{*}\right) .
\end{aligned}
$$

Thus, $Y(t)$ is in

$$
C^{\infty}(M \times\{t: t>0\}) \oplus C^{\infty}(M \times\{t: t>0\})
$$

since it is in $\bigcap_{k>0} \mathscr{D}\left(T^{2 k-q}\right)$ (resp. $\left.\bigcap_{k>q / 2} \mathscr{D}\left(T^{2 k-q}\right)\right)$, so that the first claim of the lemma follows since $V(t)$ is the first component of $Y(t)$. 
The claims for $V(t, y ; a)$ may be established just as in Kannai's proof. Indeed, if we define

$$
Y(t, y ; a)=\int_{-\infty}^{\infty} \frac{e^{-s^{2} / 4 t}}{\sqrt{4 \pi t}} \phi(s-|a|)\left[\begin{array}{c}
W(s) \\
\frac{d}{d s} W(s)
\end{array}\right] d s,
$$

then by Lemma 4 we have, for $\Omega$ a sufficiently small subset of $M$ and $\left[\begin{array}{c}W(0) \\ d W(0) / d x\end{array}\right] \in \mathscr{D}\left(T^{2 q}\right)^{*}$, that for $k$ sufficiently large that

$$
\begin{aligned}
\|V(t, y ; a)\|_{(k-q) \varepsilon, \Omega} & \leq C_{1}\left\|T^{2 k} Y(t)\right\|_{\mathscr{D}\left(T^{2 q}\right)^{*}} \\
& \leq C_{2} e^{-a^{2} / 4 t}\left\|\left[\begin{array}{c}
W(0) \\
\frac{d}{d s} W(0)
\end{array}\right]\right\|_{\mathscr{D}\left(T^{2 q}\right)^{*}}
\end{aligned}
$$

REMARK. Since $U(t)$ is a unitary group and strongly continuous on each $\mathscr{D}\left(T^{q}\right)$ and each $\mathscr{D}\left(T^{q}\right)^{*}$, the family of operators

$$
A(t)=\int_{-\infty}^{\infty}(4 \pi t)^{-1 / 2} e^{-s^{2} / 4 t} U(s) d s
$$

is in fact a (self adjoint) strongly continuous semigroup on each Hilbert space $\mathscr{D}\left(T^{q}\right)$ and $\mathscr{D}\left(T^{q}\right)^{*}$.

LEMMA 6. The support of $W(y, x, s)$ is contained in the set $\{(y, x, s)$ : $\left.s^{2}-r(y, x)^{2} \geq 0\right\}$.

Proof. A standard energy integral argument does the trick. Let $f_{1}, f_{2} \in C_{0}^{\infty}(M)$, let $u \in C^{\infty}(M \times \mathbf{R})$ satisfy

$$
\left(\frac{\partial^{2}}{\partial s^{2}}-\Delta\right) u=0, \quad u(y, 0)=f_{1}(y), \quad u_{s}(y, 0)=f_{2}
$$

We show that the support of $u$ is contained in the set

$$
V=\bigcup_{x \in \operatorname{supp}\left(f_{i}\right)}\left\{(y, s): s^{2}-r(y, x)^{2} \geq 0\right\} .
$$

Indeed, suppose $\left(y_{0}, s_{0}\right) \notin V$. Let $C$ be an open set of the form $\left\{(z, s):\left(s-s_{0}-\varepsilon\right)^{2}-r\left(z, y_{0}\right)^{2}>0,-\varepsilon<s<s_{0}+\varepsilon\right\}$. One may see that, for $\varepsilon>0$ sufficiently small, $C \cap V=\varnothing$, and that $B_{0} \cap \operatorname{supp}\left(f_{i}\right)=\varnothing$, where $B_{s}=C \cap M \times\{s\}$. If $s_{0}$ is sufficiently small, we may assume 
that $D$ is trivial on each $B_{s}$, and is spanned by $\left\{g_{a}\right\}_{a=1}^{n}$, and that $\mu$ is represented by a smooth nonzero $m$-form. Then

$$
\begin{aligned}
\frac{d}{d s} \int_{B_{s}} G^{*}(d u, d u)+\left(u_{s}\right)^{2} d \mu \\
=2 \int\left[G^{*}\left(d u, d u_{s}\right)+u_{s s} u_{s}\right] d \mu \\
\quad-\int_{\partial B_{s}}\left[G^{*}(d u, d u)+\left(u_{s}\right)^{2}\right] i\left(\frac{\partial}{\partial r}\right) d \mu \\
=2 \int_{B_{s}}\left(-\Delta u+u_{s s}\right) u_{s} d \mu-\int_{\partial B_{s}}\left[G^{*}(d u, d u)+\left(u_{s}\right)^{2}\right] i\left(\frac{\partial}{\partial r}\right) d \mu \\
\quad+2 \int_{\partial B_{s}} \sum\left(g_{a} u\right) u_{t} i\left(g_{a}\right) d \mu
\end{aligned}
$$

where $\partial / \partial r$ is the coordinate vector field associated to the spherical coordinates, $\left\{r, x_{2}, \ldots, x_{m}\right\}$, of Lemma 7 of [25]. With respect to this coordinate system

$$
\int_{\partial B_{s}} \sum\left(g_{a} u\right) u_{t} i\left(g_{a}\right) d \mu=\int_{\partial B_{s}} u_{r} u_{t} i\left(\frac{\partial}{\partial r}\right) d \mu
$$

so that

$$
\begin{aligned}
\frac{d}{d s} \int_{B_{s}} G^{*}(d \mu, d \mu)+u_{t}^{2} d \mu \\
=-\int_{\partial B_{s}} \sum_{c, i \neq 2} g_{a}^{i} g_{a}^{j} \frac{\partial u}{\partial x^{i}} \frac{\partial u}{\partial x^{j}} i\left(\frac{\partial}{\partial r}\right) d \mu \\
-\int_{\partial B_{s}}\left(u_{r}-u_{t}\right)^{2} i\left(\frac{\partial}{\partial r}\right) d \mu \leq 0,
\end{aligned}
$$

by Corollary 6 of [25]. Thus, $u \mid C=0$ since $u(y, 0) \mid B_{0}=0$. The case for $s_{0}$ arbitrary is an iteration of the above. That the quoted results of [25] are valid in this context follows from results of the next section. The result for $W$ follows from the fact that $u(y, s)=$ $\int W(y, x, s) f(x) d \mu(x)$ and $W(y, x, s)=W(x, y, s)$.

COROLlary 7. $\Delta$ is essentially self adjoint on $C_{0}^{\infty}(M)$.

Proof. It follows from the proof of the previous lemma that the function $u(y, s)$ has compact support for all $s$ by the completeness of the bicharacteristic flow of $\Delta$, and hence that $C_{0}^{\infty}(M) \oplus C_{0}^{\infty}(M)$ is invariant under $U(s)$ for all $s$. Thus, by Lemma 2.1 of Chernoff [6], $(i T)^{n}$ is essentially self adjoint for all $n>0$, hence $(i T)^{2}=\left[\begin{array}{ll}\Delta & 0 \\ 0 & \Delta\end{array}\right]$ is. 
2. Propagation of singularities. We are interested in studying the asymptotics of the fundamental solution of a hypoelliptic diffusion equation, i.e., that solution with initial condition $V(0)=\delta_{x}(=$ the $\delta$ function supported at $x \in M)$. The content of Kannai's paper is that these asymptotics are the "transmuted" version of the singularities of the solution of the wave equation with Cauchy data $W(0)=\delta_{x}$, $W_{t}(0)=0$. But the propagation of singularities theory contained in Duistermaat and Hörmander [8] may be applied to this wave equation.

In particular, let $f(y, x, t)$ denote a distribution on $M \times M \times R$, and consider the operator $\partial^{2} / \partial t^{2}-\Delta(y)$ as acting on the $y$ and $t$ variables of such distributions. Then $\left(\partial^{2} / \partial t^{2}-\Delta\right)$ has a principal symbol $h=$ $p_{t}^{2}-G^{*}(\xi, \xi)$ on $T^{*}(M \times M \times R)$, where $p_{t}$ is the dual coordinate to $t \in R$ and $\xi$ denotes an element of $T^{*} M$ (regarded as an element of the leftmost factor of $\left.T^{*}(M \times M \times R)=T^{*} M \times T^{*} M \times T^{*} R\right)$.

We then have [15] that the wave front set of $W(t, y, x)$, which we will denote by $\Lambda$, is contained in the characteristic subset $\left\{p_{t}^{2}-\right.$ $\left.G^{*}\left(\xi_{y}, \xi_{y}\right)=0\right\}$ and is invariant under the flow generated by the Hamiltonian vector field $\eta(h)$ associated with $h$. But, this flow is closely related to the singular Riemannian geodesic flow; in fact $\eta(h)=$ $2 p_{t}(\partial / \partial t)-\eta\left(G^{*}\left(\xi_{y}, \xi_{y}\right)\right)$, where $\eta\left(G^{*}(\xi, \xi)\right)$ is the Hamiltonian vector field associated with $G^{*}(\xi, \xi)$.

Now, the characteristic subset is the union of two pieces $C_{1}$ and $C_{2}$ where $C_{1}$ is the set of cotangent vectors of the form

$$
\left\{\left(\xi_{t}, \xi_{y}, \xi_{x}\right): p_{t}\left(\xi_{t}\right) \neq 0 \text { and } p_{t}\left(\xi_{t}\right)^{2}-G^{*}\left(\xi_{y}, \xi_{y}\right)=0\right\}
$$

and $C_{2}$ is the set of cotangent vectors of the form $\left\{\left(0, \xi_{y}, \xi_{x}\right): \xi_{y} \in\right.$ $\left.\operatorname{ker} G^{*}\right\} . C_{2}$ is the set of double characteristics, and on this set $\eta(h)$ vanishes since $h$ vanishes to second order.

Conversely, $\eta(h)$ does not vanish on $C_{1}$, and for $s \in R$ each submanifold $T^{*}(M \times M \times\{s\}) \cap C_{1}$ in $T^{*}(M \times M \times R) \cap C_{1}$ is a cross section for the flow generated by $\eta$. Thus, $\Lambda \cap C_{1}$ is determined by $\left.\Lambda\right|_{s=0} \cap C_{1}$. On the other hand, assuming condition (4) of the introduction, we have that $C_{2}=D^{\perp} \times T^{*} M \times T^{*} R$ is a symplectic submanifold of $T^{*}(M \times M \times R)$. This may be seen from the fact that by Darboux's Theorem for nondegenerate contact structures [1], at each $x \in M$ there are local coordinates $x^{1}, \ldots, x^{2 n}, y$ such that $\Gamma_{a}=\partial / \partial x^{a}+\sum_{b} A_{a b} x^{b}(\partial / \partial y)$, for $a=1, \ldots, 2 n$, is a local frame for $D$, where $A_{a b}$ is the antisymmetric nondegenerate form which is nonzero only on the $2 \times 2$ diagonal blocks, with each block $\left[\begin{array}{rr}0 & 1 \\ -1 & 0\end{array}\right]$. With respect to these coordinates, the principal part of $\Delta$ is a strictly positive 
quadratic form (position dependent) on the $\Gamma_{a}$. Note that this positive form is not, in general, locally diagonalizable so that $\Delta$ is not necessarily locally equivalent to the subelliptic Laplacean on a Heisenberg group. Then the Hamiltonian matrix $F_{\Delta}$ associated with the Hessian on $C_{2}$ of the principal symbol of $\Delta$ satisfies $\operatorname{ker} F_{\Delta} \cap$ Range $F_{\Delta}=0$, so that $C_{2}$ is symplectic. In this case we have the following special case of the result of Lascar and Lascar [18], which also follows from Ivrii [16].

THEOREM. The wave front set of $W$ satisfies $W F(W) \cap\{y \neq x\} \cap$ $C_{2}=\varnothing$. Thus, we may compute the wave front set away from $y=x$ simply by propagation of singularities.

Now, let $\kappa_{0}$ denote the mapping of $M \times M \rightarrow M \times M \times R$ given by $\kappa_{0}(x, y)=(x, y, 0)$. Since the pullback $\kappa_{0}^{*} W(y, x, t)=\delta_{x}(y)$ and since $\eta(h)$ is transversal to $\kappa_{0}$, it follows that

$$
\left.\Lambda \cap C_{1}\right|_{t=0}=\left\{\left(x, x, 0,-\xi, \xi, \xi_{t}\right): \xi \in T_{x}^{*} M-D^{\perp}, \xi_{t}= \pm \sqrt{G^{*}(\xi, \xi)} d t\right\}
$$

Then, since

$$
\eta(h)=2 p_{t}(0)\left(\frac{\partial}{\partial t}-\frac{1}{2 p_{t}(0)} \eta\left(G^{*}(\xi, \xi)\right)\right)
$$

we have that the wave front set of $W$ in $C_{1}$ is:

$$
\begin{array}{r}
\left\{\left(\left(y, \xi_{y}\right),\left(x, \xi_{x}\right),\left(t, \xi_{t}\right)\right)\right. \\
=\exp \left[\frac{t}{2 p_{t}(0)} \eta\left(G^{*}(\xi, \xi)\right)\right]\left(\left(x,-\xi_{x}^{0}\right),\left(x, \xi_{x}^{0}\right),\left(t, \xi_{t}^{0}\right)\right): \\
x \in M, \xi_{x}^{0} \in T_{x}^{*} M, \xi_{t}^{0}=p_{t}(0) d t, \\
\left.p_{t}(0)= \pm \sqrt{G^{*}\left(\xi_{x}^{0}, \xi_{x}^{0}\right)}\right\},
\end{array}
$$

where we regard $T^{*}(M \times M \times R)$ as identified with $\left(T^{*} M\right) \times\left(T^{*} M\right) \times$ $\left(T^{*} R\right)$. Note that $p_{t}$ is a first integral of $h$. In particular, this implies that with the exception of the set $y=x$ the singular support of $W$ is

$$
\left\{\left(\exp _{x}\left[\frac{t \xi_{x}^{0}}{2 p_{t}(0)}\right], x, t\right): \xi_{x}^{0} \in T_{x}^{*} M\right\}-\{(x, x, t): x \in M, t \in R\},
$$

where $\exp _{x}$ is the singular Riemannian exponential map discussed in [25].

To apply $\S 4$ of [25] we need to understand the limit behavior of bicharacteristics. 
Thus, let $\left\{\xi_{x}^{(n)}\right\}_{n=0}^{\infty} \subset T_{x}^{*} M$ be a sequence of cotangent vectors such that both $G_{x}^{*}\left(\xi_{x}^{(n)}, \xi_{x}^{(n)}\right)=1$ for all $n$ and $\xi_{x}^{(n)}$ goes to infinity as $n$ goes to infinity, i.e., only a finite number of the $\xi_{x}^{(n)}$ are in any compact subset of $T_{x}^{*} M$. Then, the bicharacteristic curve

$$
\begin{aligned}
\beta_{n}(s) & =\left(\left[y^{(n)}(s), \xi^{(n)}(s)\right],\left[x, \xi_{x}^{(n)}\right],\left[t=s, \xi_{t}(s)\right]\right) \\
& =\exp (s \eta(h))\left(\left(x,-\xi_{x}^{(n)}\right),\left(x, \xi_{x}^{(n)}\right),\left(0, \xi_{t}\right)\right), \quad \text { for } p_{t}\left(\xi_{t}\right)= \pm 1,
\end{aligned}
$$

is contained in $W F(W)$. Also, since $W F(W)$ is conic, the ray

$$
B_{n}(s)=\left\{\left(\left(y^{(n)}(s), \lambda \xi^{(n)}(s)\right),\left(x, \lambda \xi_{x}^{(n)}\right),\left(t, \lambda \xi_{t}(s)\right)\right): \lambda \geq 0\right\}
$$

is also in $W F(W)$. Recall now from [25] that we may (by choosing a subsequence, if necessary) assume that the geodesics $y^{(n)}(s)$ converge uniformly on compact intervals to a generalized geodesic $y_{\infty}(s)$. The corresponding sequence of $\beta_{n}(s)$ does not converge, by the completeness of $\eta(h)$, since $\xi^{(n)}(0)=-\xi_{x}^{(n)}$ goes off to infinity. However, the ray $B_{n}(s)$ has limit points for each $s$ as $n$ goes to infinity. Indeed, we may parametrize the rays by points in the cosphere bundle for some Riemannian metric tensor; since $y^{(n)}(s)$ converges, it follows that $B_{n}(s)$ is contained in a (conically) compact subset. Since $W F(W)$ is closed, it follows that these limit points are in $W F(W)$, hence, so is the ray through these limit points.

Suppose that these limit points are simply characteristic. It follows that there is a sequence $\lambda_{n}>0, \lambda_{n} \rightarrow 0$, such that

$$
\left(\left(y^{(n)}(s), \lambda_{n} \xi^{(n)}(s)\right),\left(x, \lambda_{n} \xi_{x}^{(n)}\right),\left(t, \lambda_{n} \xi_{t}(s)\right)\right)
$$

converges to a point in the simply characteristic wave front set. On the other hand, $\eta(h)$ is a complete vector field by assumption and $G^{*}(\xi, \xi)$ is a first integral, which on the characteristic variety is zero only on $C_{2}$, so it follows that $\left(G^{*}(\xi, \xi)^{-1 / 2} \eta(h)\right)$ is a complete vector field on the subset $C_{1} \subset T^{*}(M \times M \times \mathbf{R})$. Thus, $\exp \left(s\left(G^{*}(\xi, \xi)\right)^{-1 / 2} \eta(h)\right)$ is a diffeomorphism for each $s>0$, and $\Lambda \cap C_{1}$ is invariant under this diffeomorphism. This leads us to a contradiction, $\left.\Lambda \cap C_{1}\right|_{t=0}$ is an embedded submanifold of $C_{1}$ while $\left.\Lambda \cap C_{1}\right|_{t=s}$, which is the image of this set under $\exp \left(s\left(G^{*}(\xi, \xi)^{-1 / 2} \eta(h)\right)\right)$, contains the limit of the points

$$
\left(\left(y_{n}(s), \lambda_{n} \xi^{(n)}(s)\right),\left(x, \lambda_{n} \xi_{x}^{(n)}\right),\left(t=s, \lambda_{n} \xi_{t}\right)\right) .
$$

However,

$$
\left(\left(x,-\lambda_{n} \xi_{x}^{(n)}\right),\left(x, \lambda_{n} \xi_{x}^{(n)}\right),\left(0, \lambda_{n} \xi_{t}\right)\right)
$$

does not converge to a point of $\left.\Lambda \cap C_{1}\right|_{t=0}:\left.\Lambda \cap C_{1}\right|_{t=s}$ is an immersed but not an embedded submanifold. But, the diffeomorphic image of 
an embedded submanifold is embedded, so we have proven:

LEMMA 7. The limit points of the ray $B_{n}(s)$ over the generalized geodesic $y_{\infty}(s)$ are in the doubly characteristic wavefront set of $W$.

Since, as we have already discussed, the results of Lascar and Lascar [18], and Ivrii [16] give us that the doubly characteristic wavefront set of $W$ is only at $y=x$ we have

CoRollary. Any generalized geodesic $y_{\infty}(s)$ satisfies $y_{\infty}(s)=x$ for all $s$. In particular, there are no minimizing generalized geodesics in the case that $C_{2}$ is symplectic.

We may therefore apply Corollary 4 of [25], with the effect that if

$$
\begin{array}{r}
\tilde{U}=\{(y, x, t) \in M \times M \times R: y \in U(x), \\
\left.0<|t|<\mu_{x}\left(\frac{\xi(0)}{\sqrt{G^{*}(\xi(0), \xi(0))}}\right)\right\}
\end{array}
$$

where $\xi(0)$ in this case denotes $\exp _{x}^{-1} y$, where $\mu_{x}$ is a particular function on the unit sphere in $T_{x}^{*} M$ (introduced in [25]) and $U(x)$ is the dense open subset of $M$ (discussed in [25]) on which the singular Riemannian distance from $x$ to $y$ is a $C^{\infty}$ function of $y$, then sing. supp. $(W) \cap \tilde{U}=\left\{(y, x, t): r(x, y)^{2}-t^{2}=0\right\} \cap \tilde{U}$.

Even more, let $z^{1}, \ldots, z^{m}$ be the singular Riemannian geodesic spherical coordinates introduced in the proof of Lemma 7 of [25], centered at $x$ (so that $z^{1}(y)=r(y, x)$ in particular). Let $p_{1}, \ldots, p_{m}$ be the dual coordinates to $z^{1}, \ldots, z^{m}$. A consequence of Lemma 7 of [25] is the following.

LEMMA 8. Let $\pi$ denote the canonical projection of $T^{*}(M \times M \times R)$ on $M \times M \times R$. Then,

$$
\begin{array}{r}
\Lambda \cap T^{*} \tilde{U}=\left\{\left(y, x, t, \xi_{y}, \xi_{x}, \xi_{t}\right): r(y, x)^{2}-t^{2}=0, \xi_{x}=\xi_{x}(0),\right. \\
\left.\xi_{t}=p_{t}(0) d t, \xi_{y}=\operatorname{sign}(t)\left|p_{t}(0)\right| d_{y} r(y, x)\right\},
\end{array}
$$

where $d_{y}$ denotes the differential with respect to the $y$ variables.

Proof. By Corollary 6 of [25], on $\tilde{U}$,

$$
G^{*}(\xi, \xi)=p_{1}^{2}+\sum_{i, j=2}^{m} a^{i j}(y) p_{i} p_{j}
$$

for some smooth functions $a^{i j}(y)$. 
Let $s \rightarrow\left(y(s), x, t(s), \xi_{y}(s), \xi_{x}, \xi_{t}\right)$ denote the integral curve of $\eta(h)$ with initial conditions $y(0)=x, t(0)=0, \xi_{y}(0)=-\xi_{x}$. By the proof of Lemma 7 of [25] we have that $(d / d s) t(s)=p_{t}(0),(d / d s) z^{1}(y(s))=$ $2 \operatorname{sign}(s)\left|p_{t}(0)\right|$ and $(d / d s) z^{i}(y(s))=0$ for $i \geq 2$. On the other hand,

$$
\begin{aligned}
(d / d s) z^{1}(y(s)) & =\left\{z^{1}, h\right\} \quad \text { (Poisson Bracket) } \\
& =-2 p_{1}\left(\xi_{y}(s)\right)
\end{aligned}
$$

so that $p_{1}\left(\xi_{y}(s)\right)$ is independent of $s>0$ (resp. $\left.s<0\right)$. Also,

$$
\frac{d}{d s} z^{i}(y(s))=\left\{z^{i}, h\right\}=-2 \sum_{j=2}^{m} a^{i j} p_{j}\left(\xi_{y}(s)\right) .
$$

Thus, $\xi_{y}(s)=-\operatorname{sign}(s)\left|p_{t}(0)\right| d_{y} r(y, x), \bmod D^{\perp}$. Now, note that $\eta(h)$ is tangent to the submanifold of $T^{*} \tilde{U}$ given by $p_{i}=0$ for $i \geq 2$, so that if we start at some point of this submanifold and move along one of the flow lines of $\eta(h)$ for a sufficiently small distance, we will remain in this submanifold. Therefore, if we start at $(y(s), x, t(s)) \in \tilde{U}$ with a cotangent vector $\xi_{y}(s)=-\operatorname{sign}(s)\left|p_{t}(0)\right| d_{y} r(y, x), s=t(s) / p_{t}(0)$, we get that

$$
\exp [-s \eta(h)]\left(y(s), x, t(s), \xi_{y}(s), \xi_{x}, \xi_{t}\right)=\left(x, x, 0,-\xi_{x}, \xi_{x}, \xi_{t}\right),
$$

where $\xi_{x}=-\exp _{x}^{-1}(y(s))$. Indeed, on the submanifold $p_{i}=0$ for $i \geq 2$, we have $\eta(h)=p_{t}(\partial / \partial t)-p_{1}(\partial / \partial r)$. We have also

$$
\begin{aligned}
p_{1}\left(\xi_{y}(s)\right) & =-\operatorname{sign}(s)\left|p_{t}(0)\right|=-\operatorname{sign}\left(\frac{t(s)}{p_{t}(0)}\right)\left|p_{t}(0)\right| \\
& =-\operatorname{sign}(t(s)) \operatorname{sign}\left(p_{t}(0)\right)\left|p_{t}(0)\right| \\
& =-\operatorname{sign}(t(s)) p_{t}(0),
\end{aligned}
$$

so that on this submanifold, for $t>0, \eta(h)=p_{t}(0)\left(\partial / \partial t+\partial / \partial z^{1}\right)$ and for $t<0, \eta(h)=p_{t}(0)\left(\partial / \partial t-\partial / \partial z^{1}\right)$. Although $(x, x, 0)$ is not in $\tilde{U},(x, x, 0)$ is in the closure of $\tilde{U}$, and $\eta(h)$ is smooth on all of $T^{*}(M \times M \times R)$, so that we may see that, if $t=p_{t}(0) s>0$,

$$
\begin{aligned}
\exp & {[-s \eta(h)]\left(y(s), x, t(s), \xi_{y}(s), \xi_{x}, \xi_{t}\right) } \\
& =\lim _{\tilde{s} \rightarrow s} \exp \left[-\tilde{s} P_{t}(0)\left(\frac{\partial}{\partial t}+\frac{\partial}{\partial z^{1}}\right)\right]\left(y(s), x, t(s), \xi_{y}(s), \xi_{x}, \xi_{t}\right) \\
& =\left(x, x, 0,-\xi_{x}, \xi_{x}, \xi_{t}\right),
\end{aligned}
$$

by equation (2) and the definition of the singular Riemannian exponential map, since $(y(s-\tilde{s}), x, t(s-\tilde{s})) \in \tilde{U}$ for $\tilde{s}<s$. The $t<0$ case follows in the same way. 
We are now in a position to be able to say a lot about the singularities of $W$. In fact, for $(y, x, t) \in \tilde{U}$, the Hadamard construction [7, pp. 740-744] of the fundamental solution of the wave equation goes through for "hypoelliptic wave equations". We have

THEOREM 1.

$$
W(y, x, t)-\sum_{k=((1-m) / 2)}^{\infty} \delta^{(-k)}\left(t^{2}-r(x, y)^{2}\right) C_{k}(x, y) \quad \text { for }(y, x, t) \in \tilde{U}
$$

Proof. Just as in the Riemannian case $\Lambda \cap T^{*} \tilde{U}$ is the co-normal bundle of the manifold sing. supp. $(W) \cap \tilde{U}$. One can, in the usual way, identify $\left(\partial^{2} / \partial t^{2}-\Delta\right)$ with an operator on generalized $\frac{1}{2}$-densities; if the identification is via the map $f \in \mathscr{D}^{\prime}(M \times M \times R)$ goes into $f \otimes \sqrt{|d t| \otimes d \mu}$, where $d \mu$ is the smooth measure introduced in Section 1 , then the subprincipal symbol of $\left(\partial^{2} / \partial t^{2}-\Delta\right)$ is zero. In this situation, the symbol of $W \otimes \sqrt{|d t| \otimes d \mu}$ may be identified with a section, $\sigma$, of the $\frac{1}{2}$-density bundle (tensored with the Maslov bundle) on $\Lambda$ [14]. Because the subprincipal symbol of $\left(\partial^{2} / \partial t^{2}-\Delta\right)$ is zero, and the principal symbol is just the function $h$, it follows [15] that $\sigma$ is invariant with respect to the flow generated by the vector field $\eta(h)$. Thus, just as in the Riemannian case, these conditions plus the fact that $\left.W\right|_{t=0}=\delta_{x}(y)$ determine $\sigma$ completely. This is because the $\eta(h)$ flow lines in $\Lambda$ admit a global $C^{\infty}$ cross section; see Guillemin and Sternberg [12, p. 371]. Also, $\sigma$ is homogeneous under the action of the multiplicative group of the group of positive real numbers on the fibers of $T^{*}(M \times M \times R)$, because the generator of the multiplicative group action forms a two dimensional Lie algebra with $\eta(h)$ which exponentiates to a local Lie group of local diffeomorphisms; see [12].

Now, note that $\Lambda$ is diffeomorphic to the disjoint union of two copies of $R^{n}$, one copy for each choice of sign in the initial condition $\xi_{t}(0)= \pm \sqrt{G^{*}(\xi(0), \xi(0))} d t$. Denote the two components by $\Lambda^{+}$and $\Lambda^{-}$. As Duistermaat and Hörmander [8] point out, there are solutions $\psi^{+}$and $\psi^{-}$of $\left(\partial^{2} / \partial t^{2}-\Delta\right) v=0, \psi(0, y)=\delta_{x}(y)$ such that Wave Front $\left(\psi^{+}\right)=\Lambda^{+}$and Wave Front $\left(\psi^{-}\right)=\Lambda^{-}$. Guillemin and Sternberg [12, Chapter VI, Section 8] point out that $\psi^{+}$(resp. $\psi^{-}$) has Cauchy initial data $\psi^{+}(0, y)=\delta_{x}(y)$,

$$
\begin{aligned}
& \left(\frac{\partial}{\partial t} \psi^{+}\right)(0, y)=i \sqrt{-\Delta} \delta_{x}(y) \\
& \quad\left(\operatorname{resp} . \psi^{-}(0, y)=\delta_{x}(y),\left(\frac{\partial}{\partial t} \psi^{-}\right)(0, y)=-i \sqrt{-\Delta} \delta_{x}(y)\right) .
\end{aligned}
$$


Actually, Guillemin and Sternberg are concerned only with $\Delta$ elliptic on a compact manifold but, with our self adjoint $\Delta$, spectral resolutions allow us the same freedom. Thus, our solution $W$ is equal $\frac{1}{2}\left(\psi^{+}+\psi^{-}\right)$. Let $\Upsilon: M \times M \times R \rightarrow M \times M \times R$ denote the diffeomorphism $\Upsilon(y, x, t)=(y, x,-t)$, so that $\Upsilon \circ \Upsilon=\mathrm{id}$. Note that $\Upsilon$ is a symmetry of $\left(\partial^{2} / \partial t^{2}-\Delta\right)$ and that the pullback satisfies $\Upsilon^{*} \Lambda^{+}=\Lambda^{-}$. Thus, $W$ is the unique solution of the hypoelliptic wave equation with initial data $W(0, y)=\delta_{x}(y)$ such that $\Upsilon^{*} W=W$.

Now, it follows from Chapter VI, Section 4 of [12], together with Lemma 8, that the distribution

$$
u_{k}^{+}= \begin{cases}\left(t^{2}-r(y, x)^{2}+i 0\right)^{(m-1+k) / 2}, & t>0 \\ \left(t^{2}-r(y, x)^{2}-i 0\right)^{(m-1+k) / 2}, & t<0\end{cases}
$$

is a basis over the ring $C^{\infty}(\tilde{U})$ for the subspace of distributions in $I^{-1 / 4+k}\left(\tilde{U}, \Lambda^{+} \mid \tilde{U}\right)($ see $[12,14])$, with symbols which are homogeneous of degree $k$ at $\infty, \bmod C^{\infty}$. Likewise,

$$
u_{k}^{-}= \begin{cases}\left(t^{2}-r(y, x)^{2}-i 0\right)^{(m-1+k) / 2}, & t>0, \\ \left(t^{2}-r(y, x)^{2}+i 0\right)^{(m-1+k) / 2}, & t<0,\end{cases}
$$

is a basis for $I^{-1 / 4+k}\left(\tilde{U}, \Lambda^{-} \mid \tilde{U}\right)$. Thus, $\left\{u_{k}^{+}, u_{k}^{-}\right\}$is a basis for the "homogeneous" subspace of $I^{-1 / 4+k}(\tilde{U}, \Lambda \mid \tilde{U})$. Now, $\Upsilon^{*} u_{k}^{+}=u_{k}^{-}$, so that $\delta^{-(m-1+k) / 2}\left(t^{2}-r(y, x)^{2}\right)=\frac{1}{2}\left(u_{k}^{+}+u_{k}^{-}\right)$is a basis for the subspace of $I^{-1 / 4+k}(\tilde{U}, \Lambda \mid \tilde{U})$ which is homogeneous and also invariant under $\Upsilon^{*}$.

Moreover, the symbol of $\delta^{-(m-1) / 2}\left(t^{2}-r(x, y)^{2}\right)$ is nonzero on $\Lambda \cap T^{*} \tilde{U}$ so that there is a smooth function $C_{(m-1) / 2}(x, y)$ of $x$ and $y$ such that $\delta^{-(m-1) / 2}\left(t^{2}-r(x, y)^{2}\right) C_{(m-1) / 2}(x, y)$ has the same symbol as $W$. Note that $|t|=r(x, y)$ on $\Lambda$ and that $W(y, x, t)=W(y, x,-t)$ implies that $W$ depends only on $|t|$; thus $C_{(m-1) / 2}(x, y)$ need have no $t$ dependence. $C_{(m-1) / 2}(x, y)$ may be computed as the solution of a linear first order partial differential equation, a "transport equation", on $\Lambda \cap T^{*} \tilde{U}$, just as in the Riemannian situation discussed by Kannai [17]. Thus, since both $W$ and $\delta^{-(m-1) / 2}\left(t^{2}-r(x, y)^{2}\right)$ belong to $I^{-1 / 4}(\tilde{U}, \Lambda \mid U)$ (in the notation of Hörmander), it follows that $W-\delta^{-(m-1) / 2}\left(t^{2}-r(x, y)^{2}\right) C_{(m-1) / 2}(x, y) \in I^{3 / 4}(\tilde{U}, \Lambda \mid \tilde{U})$. But, $\delta^{-(m+1) / 2}\left(t^{2}-r(x, y)^{2}\right) \in I^{3 / 4}(\tilde{U}, \Lambda \mid \tilde{U})$, and has a symbol which is nonzero, of the same homogeneity as the symbol of $W-\delta^{-(m-1) / 2}\left(t^{2}-r(x, y)^{2}\right) C_{(m-1) / 2}(x, y)$ and invariant under $\Upsilon$. Thus, there is a smooth function $C_{(m+1) / 2}(x, y)$, which may be computed as 
the solution of a transport equation, such that

$$
\begin{aligned}
W & -\delta^{-(m-1) / 2}\left(t^{2}-r(x, y)^{2}\right) C_{(m-1) / 2}(x, y) \\
& -\delta^{-(m+1) / 2}\left(t^{2}-r(x, y)^{2}\right) C_{(m+1) / 2}(x, y) \in I^{1 \frac{3}{4}}(\tilde{U}, \Lambda \mid \tilde{U}) .
\end{aligned}
$$

In this way, the functions $C_{k}(x, y)$ may be computed (recursively: $C_{k+1}$ depends on $C_{k}$ ) in such a way that

$$
W-\sum_{k=(1-m) / 2}^{N} \delta^{(-k)}\left(t^{2}-r(x, y)^{2}\right) C_{k}(x, y) \in I^{N+m-1 / 4}(\tilde{U}, \Lambda \mid \tilde{U}) .
$$

But, $N$ may be chosen so that the elements of this space are of an arbitrary specified degree of smoothness.

We now apply the previous results to the consideration of the hypoelliptic diffusion equation $(\partial / \partial t-\Delta) \rho(y, x, t)=0, \lim _{t \rightarrow 0} \rho(y, x, t)=$ $\delta_{x}(y)$.

We have:

TheOREM 2. Suppose $(y, x, s) \in \tilde{U}$ for some $s \neq 0$. Then

$$
\rho(t, y, x) \sim \frac{1}{(4 \pi t)^{m / 2}} e^{-r(y, x)^{2} / 4 t} \sum_{j=0}^{\infty} v_{j}(y, x) t^{j},
$$

where each $v_{j}(y, x)$ is a smooth function on $M \times M$.

Proof. We have that the support $\operatorname{supp}(W) \subseteq\left\{(y, x, t): r(y, x) \leq t^{2}\right\}$, just as in the Riemannian case. The proof is then exactly as that for the Riemannian case (i.e., parabolic diffusion equations) as discussed in $\S 3$ of Kannai's paper [17], with his Lemma 2.1 replaced by Lemma 5; the only difference is that here the set $U(x)=\{y:(y, x, t) \in \tilde{U}$ for some $t$ \} does not contain a neighborhood of $x$ in $M$.

REMARKS. (1) Note that hypoellipticity implies that the non-smooth behavior of $\rho$ is located at $t=0, x=y$. But $(x, x, s)$ is not in $\tilde{U}$ for any $s$, since by considerations of [25], $r(x, y)$ is not smooth at $x=y$. (2) Note the somewhat surprising fact that for both the situation we discuss in Theorem 2 and the case that $\Delta$ is elliptic (for each appropriately chosen $(x, y)) \rho(t, x, y) \sim c t^{-m / 2} e^{-K / t}$ for suitable $C, K>0$. This is in marked contrast with the situation on the diagonal $x=y$ (see [19], for example).

We have the following corollary. 
COROllaRy 8. $\lim _{t \rightarrow 0}-4 t \ln \rho(y, x, t)=r(y, x)^{2}$.

Proof. The proof is easy if $(y, x, s) \in \tilde{U}$ for some $s \neq 0$, since by the previous theorem,

$$
(4 \pi t)^{m / 2} \rho(t, x, y) \exp \left[\frac{r(x, y)^{2}}{4 t}\right] \sim \sum_{j=0}^{\infty} v_{j}(x, y) t^{j}
$$

Hypoellipticity implies that $\rho$ is $C^{\infty}$ for $t>0$, and $r(y, x)^{2}$ is $C^{\infty}$ as long as $(y, x, s) \in \tilde{U}$ for some $s \neq 0$, so that for $t>0$,

$$
\rho(y, x, t)=(4 \pi t)^{-m / 2} \exp \left[-\frac{r(x, y)^{2}}{4 t}\right] v(x, y, t)
$$

where $v(x, y, t)$ is a $C^{\infty}$ function of all variables, which is nonzero for $t>0$, which may be extended to a $C^{\infty}$ function of $t \in \mathbf{R}$ and which satisfies $v(y, x, t) \sim \sum_{j=0}^{\infty} v_{j}(x, y) t^{j}$ at $t=0$. The sought after limit then follows uniformly for $(y, x)$ contained in compact subsets of $\{(y, x):(y, x, s) \in \tilde{U}$ for some $s\} M \times M$. As Kannai points out, the inequality

$$
\liminf _{t \rightarrow 0}-4 t \ln \rho(y, x, t) \geq r(y, x)^{2}
$$

follows from the fact that supp $W(y, x, s)\left\{(y, x, s): s^{2}-r(y, x)^{2} \geq 0\right\}$ and the transmutation formula (this inequality was first proved by Azencott [2] using large deviation methods). This formula is proved by using Lemma 5 to show that $\rho(y, x, t)=O\left(e^{\left(r(x, y)^{2}-\varepsilon\right) / 4 t}\right)$ uniformly for $(y, x)$ in compact subsets of $M \times M$. One can also demonstrate the inequality

$$
\limsup _{t \rightarrow 0}-4 t \ln \rho(y, x, t) \leq r(y, x)^{2}
$$

uniformly on compacta in $M \times M$. The proof is exactly that of Theorem 4.9 of Kannai, with a little care taken, Corollary 7 of [25] guarantees the existence of open neighborhoods of any point on which Theorem 1 holds, and here $r(x, y)$ is equal to Kannai's $e(x, y)$.

Note added in proof. Results independently obtained which are related to the results of this paper are the following. The author gratefully acknowledges discussions with and/or manuscripts from the following authors.

R. Léandre, Minoration en temps petit de la densité d'une diffusion dégénérée, J. Funct. Anal., 74 (1987), 399-414. 
R. Melrose, Propagation for the wave group of a positive subelliptic second order differential operator, in 'Hyperbolic Equations and Related Topics,' ed. S. Mizohata, Academic Press (1986).

S. Watanabe, Analysis of Wiener functionals (Malliavin calculus) and its applications to heat kernels, Ann. Prob., 15 (1988), 1.

\section{REFERENCES}

[1] V. I. Arnold, Mathematical Methods of Classical Mechanics, Springer-Verlag, New York (1978).

[2] R. Azencott, Géodesiques et Diffusions en Temps Petit, Astérisque 84-85, Société Mathématique de France.

[3] J. M. Bismut, Large Deviations and the Malliavin Calculus, Birkhäuser, Boston (1984).

[4] R. Brockett, Control Theory and Singular Riemannian Geometry, in New Directions in Applied Mathematics, Springer-Verlag, New York (1981).

[5] J. Cheeger, M. Gromov, and M. Taylor, Finite propagation speed, kernel estimates for functions of the Laplace operator and the geometry of complete Riemannian manifolds, J. Diff. Geom., 17 (1982), 15.

[6] P. R. Chernoff, Essential self adjointness of powers of generators of hyperbolic equations, J. Funct. Anal., 12 (1973), 401-414.

[7] Courant and Hilbert, Methods of Mathematical Physics, Interscience (1962).

[8] J. J. Duistermaat and L. Hörmander, Fourier Integral Operators. II, Acta Math., 128 (1972), 183.

[9] C. Fefferman and D. H. Phong, Subelliptic Eigenvalue Problems, in Proceedings of the conference on harmonic analysis in honor of Antoni Zygmund, Wadsworth Math. Series 1981, p. 590.

[10] B. Gaveau, Principe de moindre action, propagation de la chaleur et estimées sous elliptiques sur certains groupes nilpotents, Acta Math., 139 (1977), 95.

[11] M. Gromov, Sur structures metriques pour les varietes Riemanniens, CedicNathan 1981.

[12] V. Guillemin and S. Sternberg, Geometric Asymptotics, Mathematical Surveys No. 14, Amer. Math. Soc. (1977).

[13] L. Hörmander, Hypoelliptic second order differential equations, Acta Math., 119 (1967), 147.

[14] _ Fourier integral operators I, Acta Math., 127 (1971), 79.

[15] _ Spectral Analysis of Singularities, in Seminar on Singularities of Solutions of Linear Partial Differential Equations, Lars Hörmander, ed., Annals of Mathematics Studies, No. 91, Princeton University Press (1979).

[16] V. Ja. Ivrii, Wave fronts of solutions of certain hyperbolic pseudodifferential equations, Trans. Moscow Math. Soc., 39 (1981), 87-119.

[17] Y. Kannai, Off diagonal short time asymptotics for fundamental solutions of diffusion equations, Comm. in P.D.E., 2(8), (1977), 781.

[18] B. Lascar and R. Lascar, Propagation des Singularités pour des Equations $\mathrm{Hy}$ perboliques à Caractéristiques de Multiplicité au Plus Double et Singularités Masloviennes II, J. d'Analyse Math., 41 (1982), 1.

[19] A. Menikoff and J. Sjostrand, On the eigenvalues of a class of hypoelliptic operators, Math. Ann., 235 (1978), 55-85. 
[20] L. P. Rothschild and E. M. Stein, Hypoelliptic differential operators and nilpotent groups, Acta Math., 137 (1977), 247.

[21] A. Sanchez-Calle, Fundamental solutions and geometry of the sum of squares of vector fields, Invent. Math., 78 (1984), 143.

[22] R. S. Strichartz, Sub-Riemannian geometry, J. Diff. Geom., 24 (1986), 221-263.

[23] M. Taylor, Noncommutative Microlocal Analysis, Part I, Memoirs AMS No. 313.

[24] T. J. S. Taylor, A parametrix for step-two hypoelliptic diffusion equations, Trans. Amer. Math. Soc., 296 (1986), 191-215.

[25] - Some aspects of differential geometry associated with hypoelliptic second order operators, Pacific J. Math., 136 (1989), 355-378.

[26] S. R. S. Varadhan, On the behavior of the fundamental solution of the heat equation with variable coefficients, Comm. Pure Appl. Math., 20 (1967), 431.

Received November 24, 1986 and in revised form July 28, 1987. This work was supported in part by the Office of Naval Research under JSEP contract number N004175-C-0648 and in part by Arizona State University through a Faculty Grant in Aid.

ARIZONA State University

TEMPE, AZ 85287 



\section{PACIFIC JOURNAL OF MATHEMATICS \\ EDITORS}

\author{
V. S. VARADARAJAN \\ (Managing Editor) \\ University of California \\ Los Angeles, CA 90024 \\ HERBERT ClEMENS \\ University of Utah \\ Salt Lake City, UT 84112 \\ THOMAS ENRIGHT \\ University of California, San Diego \\ La Jolla, CA 92093
}

R. FINN

Stanford University

Stanford, CA 94305

HERMANN FLASCHKA

University of Arizona

Tucson, AZ 85721

VAUGhan F. R. JONES

University of California

Berkeley, CA 94720

STEVEN KERCKHOFF

Stanford University

Stanford, CA 94305

\section{ROBION KIRBY}

University of California

Berkeley, CA 94720

C. C. MOORE

University of California

Berkeley, CA 94720

HAROLD STARK

University of California, San Diego

La Jolla, CA 92093

\section{ASSOCIATE EDITORS}
R. ARENS
E. F. BECKENBACH
B. H. NEUMANN
F. WOLF
K. YOSHIDA

(1906-1982)

\section{SUPPORTING INSTITUTIONS}

UNIVERSITY OF ARIZONA

UNIVERSITY OF BRITISH COLUMBIA

CALIFORNIA INSTITUTE OF TECHNOLOGY

UNIVERSITY OF CALIFORNIA

MONTANA STATE UNIVERSITY

UNIVERSITY OF NEVADA, RENO

NEW MEXICO STATE UNIVERSITY

OREGON STATE UNIVERSITY

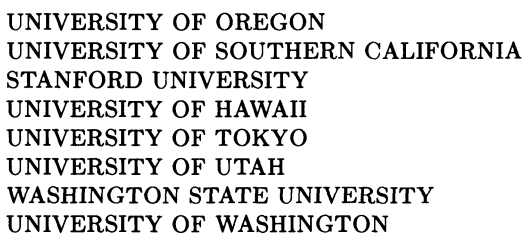

The Supporting Institutions listed above contribute to the cost of publication of this Journal, but they are not owners or publishers and have no responsibility for its content or policies.

\footnotetext{
Mathematical papers intended for publication in the Pacific Journal of Mathematics should be in typed form or offset-reproduced (not dittoed), double spaced with large margins. Please do not use built up fractions in the text of the manuscript. However, you may use them in the displayed equations. Underline Greek letters in red, German in green, and script in blue. The first paragraph must be capable of being used separately as a synopsis of the entire paper. In particular it should contain no bibliographic references. Please propose a heading for the odd numbered pages of less than 35 characters. Manuscripts, in triplicate, may be sent to any one of the editors. Please classify according to the scheme of Math. Reviews, Index to Vol. 39. Supply name and address of author to whom proofs should be sent. All other communications should be addressed to the managing editor, or Elaine Barth, University of California, Los Angeles, California 90024.

There are page-charges associated with articles appearing in the Pacific Journal of Mathematics. These charges are expected to be paid by the author's University, Government Agency or Company. If the author or authors do not have access to such Institutional support these charges are waived. Single authors will receive 50 free reprints; joint authors will receive a total of 100 free reprints. Additional copies may be obtained at cost in multiples of 50 .
}

The Pacific Journal of Mathematics is issued monthly as of January 1966. Regular subscription rate: $\$ 190.00$ a year (5 Vols., 10 issues). Special rate: $\$ 95.00$ a year to individual members of supporting institutions.

Subscriptions, orders for numbers issued in the last three calendar years, and changes of address should be sent to Pacific Journal of Mathematics, P.O. Box 969, Carmel Valley, CA 93924, U.S.A. Old back numbers obtainable from Kraus Periodicals Co., Route 100, Millwood, NY 10546.

The Pacific Journal of Mathematics at P.O. Box 969, Carmel Valley, CA 93924 (ISSN 0030-8730) publishes 5 volumes per year. Application to mail at Second-class postage rates is pending at Carmel Valley, California, and additional mailing offices. Postmaster: send address changes to Pacific Journal of Mathematics, P.O. Box 969, Carmel Valley, CA 93924.

PUBLISHED BY PACIFIC JOURNAL OF MATHEMATICS, A NON-PROFIT CORPORATION Copyright (C) 1989 by Pacific Journal of Mathematics 


\section{Pacific Journal of Mathematics}

\section{Vol. 136, No. 2 December, 1989}

A. K. Agarwal and David Bressoud, Lattice paths and multiple basic hypergeometric series ................................. 209

Adalberto Panobianco Bergamasco and Hermano de Souza Ribeiro, Uniqueness in a doubly characteristic Cauchy problem ........... 229

Thomas Curtis Craven and George Leslie Csordas, Jensen polynomials

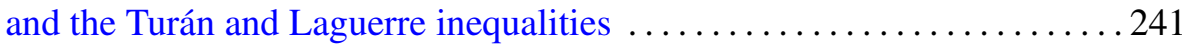

Gary R. Jensen and Marco Rigoli, Harmonic Gauss maps ............ 261

L. G. Kovács and Cheryl Elisabeth Praeger, Finite permutation groups with large abelian quotients $\ldots \ldots \ldots \ldots \ldots \ldots \ldots \ldots \ldots \ldots \ldots \ldots . \ldots \ldots$

Ken-ichi Maruyama, Localization of a certain subgroup of self-homotopy

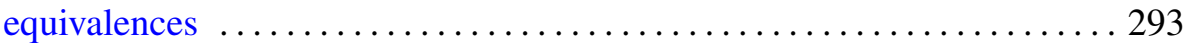

Tomasz Mazur, Canonical isometry on weighted Bergman spaces ......... 303

Bernt Karsten Oksendal, A stochastic Fatou theorem for quasiregular

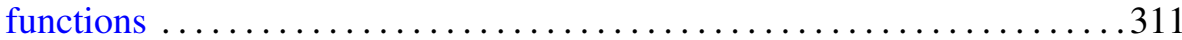

Ian Fraser Putnam, The $C^{*}$-algebras associated with minimal homeomorphisms of the Cantor set ........................ 329

Tom Joseph Taylor, Some aspects of differential geometry associated with

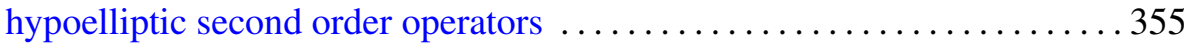

Tom Joseph Taylor, Off diagonal asymptotics of hypoelliptic diffusion equations and singular Riemannian geometry 